\title{
¿Cómo transformar ejercicios tradicionales de libros en experiencias comunicativas?
}

\author{
Por: Mag. Luis Diego Guillén Jiménez', Instituto Tecnológico de Costa Rica \\ Recibido: $\quad 8$ marzo, 2017. \\ Aprobado: 29 mayo, 2017.
}

\section{Resumen}

Las perspectivas modernas en la enseñanza de segundas lenguas apuntan a un sistema en el que la comunicación es más importante que el conocimiento metalingüístico (Cirocki y Widodo, 2012). Por años, las investigaciones sobre los enfoques comunicativos han demostrado que una enseñanza basada en competencias y habilidades comunicativas es mucho más efectiva para la adquisición de una segunda lengua o lengua extranjera en comparación con los métodos tradicionales (Nunan, 1989, 2004; Lee, 2000; Richards y Rodgers, 2001; Ellis, 2003; Hernández, 2006). A pesar de esto, muchos textos usados por los docentes ofrecen pocos ejercicios o prácticas con este enfoque. Esto obliga a los educadores a dedicar tiempo y esfuerzo en buscar o diseñar materiales alternativos al libro de texto, mientras este se desaprovecha. Este artículo propone un proceso creativo para adaptar los ejercicios de los libros de texto y transformarlos en tareas comunicativas (Ellis, 2009). Para ello, se parte de algunos principios del Aprendizaje por Tareas (Ellis, 2003; Willis \& Willis, 2012) y se presenta un ejemplo concreto del método aplicado en un ejercicio tradicional. Dado que este es un ejercicio de creatividad (Xerri y Vassallo, 2016), no se pretende ofrecer tipologías de tareas, sino pasos en el proceso de transformación que surgen de la experiencia del autor y de los principios del enfoque de Aprendizaje por Tareas.

\section{Abstract \\ How to transform traditional book exercises into communicative experiences?}

Modern perspectives in second language teaching seek for a system in which communication is more important than metalinguistic knowledge (Cirocki \& Widodo, 2012). Through years, research on communicative approaches had pointed out that teaching based on competencies and communicative skills is much more effective for second or foreign language acquisition than traditional methods (Nunan 1989, 2004; Lee, 2000; Richards \& Rodgers, 2001; Ellis, 2003, Hernández, 2006). Despite this, many text books used by teachers offer few exercises and activities with this approach. This forces educators to spend time and effort in the search for or design of alternative materials to the textbook, while this is poorly used. This article puts forth a creative process to adapt textbook exercises into communicative tasks (Ellis, 2009). In order to do so, some Task Based principles (Ellis, 2003; Willis \& Willis, 2012) are presented along with a concrete example of the method applied to a sample traditional exercise. Since this is a creative practice (Xerri \& Vassallo, 2016), it is not the aim to present taxonomies of tasks, but the steps in the transformation process that come from the author's experience and the principles of Task Based Instruction.

1 Luis Diego Guillén es máster en Inglés para Propósitos Específicos, graduado de la Universidad de Costa Rica. Actualmente se desempeña como docente en el Instituto Tecnológico de Costa Rica. Contacto: Iguillen@itcr.ac.cr.
Luis Diego Guillén Jiménez. ¿Cómo transformar ejercicios tradicionales de libros en experiencias comunicativas? Revista Comunicación. Año 38, volumen 26, número 1, enero - junio, 2017. Instituto Tecnológico de Costa Rica. ISSN: 0379-3974 / e-ISSN: 16593820 .

\section{PALABRAS CLAVE:}

Aprendizaje por tareas, enfoque comunicativo, aprendizaje de segunda lengua, lengua extranjera, metodología, creatividad.

\section{KEY WORDS:}

Task based learning, communicative approach, second language learning, foreign language, methodology, creativity. 


\section{INTRODUCCIÓN}

En el ámbito de la enseñanza de lenguas extrajeras o segundas lenguas, los docentes están en permanente búsqueda de actividades que promuevan la comunicación, pues se ha demostrado que este tipo de interacciones en clase favorecen la adquisición de una lengua, a la vez que ayudan a mantener un buen ambiente para el aprendizaje (Robertson, 2014; WiIlis \& Willis, 2012; Nunan, 1989, 2004; Lee, 2000; Richards y Rodgers, 2001; Ellis, 2003; Hernández, 2006). Esta tarea no es fácil de conseguir ya que los programas de estudio de muchas instituciones usualmente favorecen el uso de un libro como material didáctico, el cual, desafortunadamente, no siempre ofrece el tipo de ejercicio necesario para llevar a cabo estos actos comunicativos. Ante este problema, se suele argumentar que el libro es una guía y que el docente no debe basarse en él sino adaptar lo que le sirva y complementar con otros materiales y actividades. Esta respuesta no es muy satisfactoria dado el alto valor económico de los libros de texto. Además, la búsqueda de material extra o el diseño de materiales y actividades por parte del docente es demandante en tiempo y a veces dinero. Existe, sin embargo, otra opción: transformar los ejercicios tradicionales presentes en estos libros en actividades comunicativas, que cumplan más con los estándares de calidad modernos. Para ello, el presente artículo propone un método de creación de actividades comunicativas que parte de los ejercicios tradicionales de los libros de texto. En una primera instancia, se ofrece el marco teórico del enfoque de Aprendizaje por Tareas (APT) ya que, pesar de estar en una época postmétodo (Cirocki y Widodo, 2012), el APT se mantiene como una de las formas más recomendadas por los expertos (Hernández, 2006) para organizar y guiar las clases. Su principal característica es el concepto de tarea el cual será definido en la primera parte de este artículo. Luego, se presenta una revisión de los tipos de tareas de acuerdo con las diferentes perspectivas dadas por los autores más consolidados en el tema. Una vez claros estos conceptos, se ofrece, mediante el análisis de un ejercicio tradicional, la metodología de pasos para la transformación de ejercicios en tareas. Se da énfasis en el hecho de que no es una receta de cómo se debe hacer, sino unos pasos que surgen desde la experiencia del autor y los principios del APT; se ofrecen como punto de par- tida para una mayor discusión del tema. Por último, se reproducen un listado de criterios para evaluar la calidad de las tareas diseñadas.

\section{¿QUÉ ES UNA TAREA?}

Dentro del aprendizaje de una segunda lengua, el TBI (Task-Based Instruction, por sus siglas en inglés) o APT en español, surge como un enfoque comunicativo que hace uso del concepto de tarea (task) como medio idóneo para facilitar la adquisición de una segunda lengua. Si bien es cierto, este enfoque ha sido utilizado en la enseñanza del español (véase por ejemplo, Martín, 2009; Zanón, 1995; Lázaro, 1999; Fernández y Navarro, 2010), su mayor desarrollo y estudio se ha dado en la enseñanza del inglés (Ellis, 2003; Lee, 2000; Nunan, 1989, 2004 y Willis \& WiIlis, 2012), además de innumerables publicaciones de estudios realizados alrededor del mundo).

El concepto de tarea ha tenido diversas definiciones según la perspectiva de cada autor. Así por ejemplo, una de las más recientes la encontramos en Fernández y Navarro (2010) que las define como:

Actividades concretas, reales o verosímiles, acciones de la vida cotidiana -no sólo académicas-, de interés para los alumnos, que se realizan en clase, o desde la clase, y que como ejes de la unidad didáctica, dinamizan los procesos de comunicación y de aprendizaje y llevan a aprender todo lo necesario para poder realizarlas en la nueva lengua (p. 7).

Por su parte, Nunan (1989) posee una de las definiciones clásicas en la que una tarea es:

...una pieza del trabajo en clase que involucra a los alumnos en comprender, manipular, producir o interactuar en la lengua meta mientras que su atención está enfocada en movilizar su conocimiento gramatical con el fin de expresar significado, y en el que la atención está en expresar significado más que en manipular la forma. La tarea debe además tener un sentido de completud, ser capaz de mantenerse como un acto comunicativo en sí mismo con un principio un medio y un final (p. 10).

Aparte de las leves diferencias en las definiciones, pues la primera abarca desde la tarea en clase como 


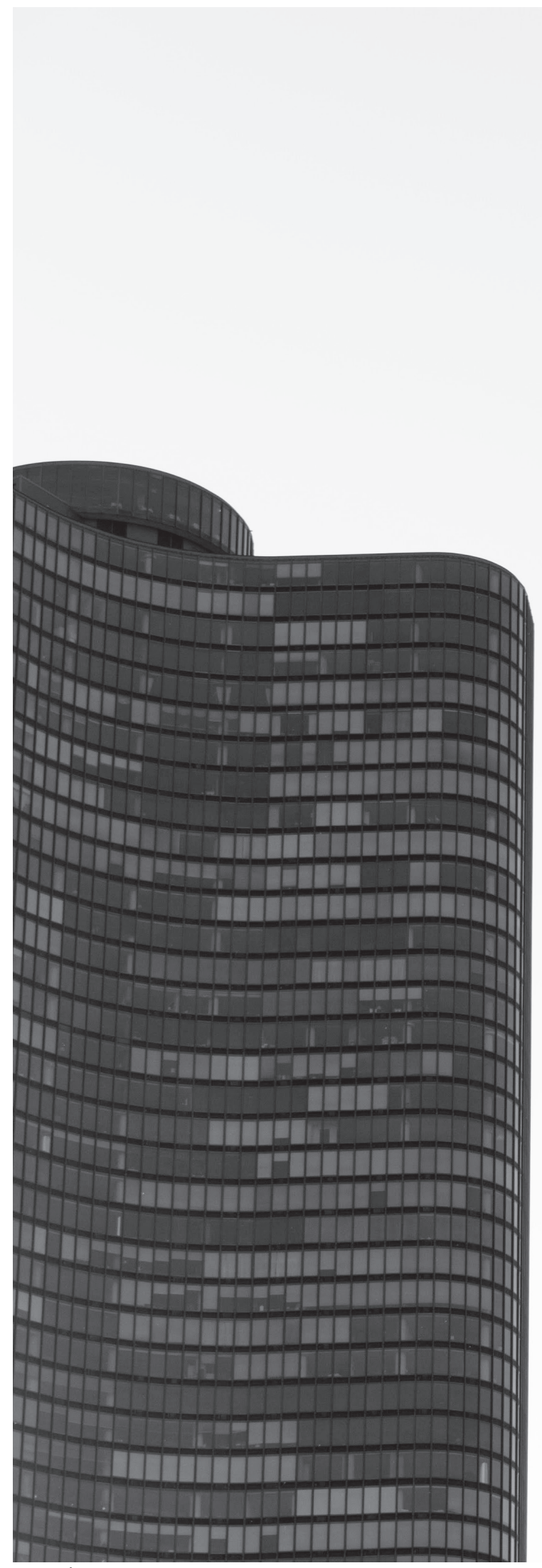

Fotografía de Leonardo Corrales N. un ente dinamizador de los procesos comunicativos $y$, por tanto, del aprendizaje de una lengua, hasta su inserción como eje de unidad temática; mientras la segunda definición se enfoca más en la idea de una tarea como un acto comunicativo especifico con una estructura clara enfocada en el contenido y no en la forma. De esta manera se puede asegurar que existe "un entendimiento esencial de que una tarea es una actividad u objetivo que es llevado a cabo por medio del lenguaje." (Richards y Rodgers, 2001, p. 224). Así pues, una tarea se basa en el contenido y utiliza el lenguaje como medio para la comunicación. Por su parte, se suele entender que un ejercicio se enfoca en la forma y no necesariamente sirve para un acto comunicativo sino para la consolidación de algún tipo de conocimiento lingüístico, llámese gramática, léxico, sintaxis, etc. (Robertson, 2014; Willis \& WiIlis, 2012, Martín, 2009; Hernández, 2006; Nunan, 2004; Ellis, 2003; Lee, 2000 y Zanón, 1995).

Para los propósitos del presente trabajo es importante resaltar la diferencia entre ejercicios y tareas. Para esto, Nunan (2004) propone prestar atención a los resultados esperados. Por un lado, un ejercicio busca responder a la pregunta: “ ¿Usó el alumno el lenguaje de manera correcta?". Por el otro, una tarea responde a la pregunta: "¿Pudo el alumno seleccionar el tipo de ropa adecuada de acuerdo al reporte del clima?" (p. 23). Como se ve, estas preguntas responden a ese contraste entre forma y contenido. Así pues, un ejercicio tiene un límite lingüístico dentro del contexto del aula o del libro de texto mientras que una tarea está más en contacto con la realidad cotidiana y no se limita al aula, aunque se lleve a cabo allí. Un alumno no va a realizar ejercicios de pareo sobre el presente perfecto como parte de su cotidianidad, en su trabajo o con sus amigos, pero es claro que necesitará llevar a cabo interacciones como la del ejemplo dado por Nunan (2004). Además, un ejercicio no pasa de ser una práctica puntual que a veces cae en la repetición, por ejemplo: respuesta múltiple, completar espacios, reordenar oraciones, entre otros; a diferencia de una tarea que implica creatividad y negociación, por ejemplo: actividades de vacío de información.

Vale destacar en este punto, ya que usualmente es motivo de confusión, que el APT no descuida los aspectos formales o la corrección a la hora del acto 
comunicativo, sino que los ubica en momentos que no interrumpan dicho proceso (sobre los malos entendidos del enfoque véase: Ellis, 2009).

\section{TIPOS DE TAREAS}

Una vez clara la diferencia entre una tarea y un ejercicio, es importante revisar las tipologías, características y ejemplos de las tareas. Nunan (2004) distingue dos tipos de tareas. Aquellas que se dan en el mundo real (real-world or target tasks) que son actividades realizadas fuera del aula como parte de nuestro día a día, y las tareas pedagógicas, que se dan en el contexto educativo. Estas últimas a su vez se dividen en dos tipos: tareas de ensayo y tareas de activación. Las tareas de ensayo emulan directamente el mundo real. Por ejemplo, en nuestra cotidianidad enviamos correos electrónicos, hacemos llamadas, preparamos reportes, vemos las noticias, etc. Este tipo de tareas pueden ser reproducidas en el aula sin mayores problemas. En cuanto a las tareas de activación son aquellas que no esperaríamos en una situación de la vida real pero que activan habilidades comunicativas transferibles a situaciones reales. Por ejemplo, es muy poco probable que un alumno deba resolver alguna escena de un crimen o dar una conferencia sobre el cambio climático en un foro mundial (a menos que sean detectives o especialistas en el área, claro está). Sin embargo, este tipo de actividades les permite practicar, de manera amena, habilidades comunicativas requeridas con frecuencia: describir un lugar, persona o situación y dar su opinión de manera estructurada e informada sobre diversos temas.

$\mathrm{Al}$ revisar lo escrito sobre el APT, hay una variedad de tipologías de las tareas, muchas de las cuales son referencias a trabajos seminales. Richard y Rodgers (2001) explican que "hay muchas descripciones en competencia de tipos básico de tareas en APT y de actividades de clase apropiadas" (p. 233). Estos autores rescatan los trabajos de Pica, Kanagy y Falodun (1993) y Willis (1996) por ser los más ampliamente reproducidos y funcionales.

Pica, Kanagy y Falodun (1993) propusieron una clasificación basada en el tipo de interacción que ocurre mientras se realiza la actividad. En ese sentido, hay tareas de: rompecabezas, vacío de información, problema-solución, toma de decisiones e intercambio de opinión. De manera similar, Willis (1996) planteó una de las más populares y reproducidas. En esta, se habla de seis etiquetas para las tareas entendidas como actividades realizadas por los alumnos: listado, ordenamiento y escogencia, comparación, resolución de problemas, compartir experiencias personales y tareas creativas. Esta última incluye actividades artísticas o las que requieran un proceso creativo de algún tipo.

Por su parte, Ellis (2003) propone ver dos tipos básicos de tareas dependientes del manejo del lenguaje: las tareas desenfocadas donde los alumnos no deben usar un tipo determinado de lenguaje; y las tareas enfocadas, que pretenden inducir a los alumnos a usar un aspecto determinado del lenguaje estudiado.

Finalmente, Nunan (2004) propone tres tipos básicos de tareas que parten del concepto de "gap" o vacío. Estas serían:

\section{1. vacío de información,}

2. vacío de razonamiento,

3. vacío de opinión.

La palabra "vacío" se emplea pues se entiende que la comunicación es un tipo de negociación de significados en la cual un participante tiene la información, razonamiento u opinión que el otro requiere y por tanto, se da el acto comunicativo para llenar ese vacío. Todas estas descripciones responden simplemente a la variedad de enfoques para analizar cualidades o características de las tareas o de la acción en que se involucra al alumno como medio para adquirir un lenguaje. Es importante considerarlas como guías a la hora de diseñar una tarea ya que algunas de ellas categorizan mientras otras diversifican las posibilidades. Sin embargo, en sí mismas no son tareas, y entender estas descripciones no es suficiente para poder diseñar una tarea a partir de los ejercicios de un libro de texto. Para ello, es esencial tener un proceso de diseño, explicado en la siguiente sección.

\section{DISEÑO DE UNA TAREA}

Al proponer cómo diseñar tareas, no es suficiente con listar posibles actividades (Fernández y Navarro, 2010) pues esto no ayuda al proceso creativo ni de adaptación que los docentes deben llevar a cabo. En otras palabras, se cae en el riesgo de que los educadores plagien las tareas propuestas sin tener presente sus contextos y sus objetivos. Asimismo, las diversas 
tipologías mencionadas no son en sí mismas ejemplos de tareas, sino de los proceso de comunicación que diversas tareas pueden o deben llevar a cabo. En vez de eso, y como parte del movimiento post-método (Cirocki y Widodo, 2012), es importante estimular algún proceso creativo (Xerri y Vassallo, 2016) que le permita a los docentes, desde su contexto, estructurar tareas acordes con las necesidades de sus alumnos, su nivel, sus intereses, los objetivos del currículo y del material usado.

Por ello, lejos de ofrecer un listado de actividades por hacer, se plantea a continuación un proceso de creación de tareas que dé las pautas y marque los límites deseables de una tarea, sin ofrecer una "receta". Dado que la mayoría de los profesores usan libros de texto llenos de ejercicios, la propuesta parte de transformar esos ejercicios en tareas comunicativas. Es importante recalcar que no es aconsejable transformar todos los ejercicios de un libro de texto a tareas comunicativas, ya que los ejercicios tienen su validez como refuerzo y consolidación de conocimientos lingüísticos sin los cuales la comunicación perdería asertividad (Robertson, 2014). No obstante, partir de los ejercicios en un libro de texto es una forma simple de crear tareas con un uso creativo de los recursos que se tienen a mano en los contextos educativos particulares.

Para el proceso creativo se plantean cinco pasos con sus respectivas preguntas guía. Dichos pasos fueron concebidos a través de la experiencia personal del autor y se basan en la teoría propuesta por Ellis (2003) quien establece un marco de características a tomar en cuenta cuando se crea una tarea. El primer paso es establecer el objetivo que les indica a los alumnos qué hacer y cuándo finalizó la tarea. Debe además ser un objetivo comunicativo y no lingüístico. El segundo paso es el "input" o la información necesaria para entender lo que debe hacer: esto se refiere a los ejemplos o ideas ofrecidas a los alumnos como modelos o como aspectos contextualizadores que les permitan entender el marco en el cual se realiza la comunicación. El tercer paso es señalar las condiciones, y esto responde a la pregunta de cómo se presenta la información. El cuarto es el proceso o reglas de cómo desarrollar la actividad. Y por último los resultados esperables (producto o proceso): comprar algo, llegar a un acuerdo, levantar una lista, etc.
Para que los pasos de creación de tareas sean fáciles de entender, se presenta en la figura 1 un ejemplo de ejercicio tradicional de libro el cual servirá de guía para ilustrar el proceso de análisis.

La Figura 1 presenta un ejercicio no comunicativo en el que se le pide a los alumnos escribir oraciones del estilo "me gusta el inglés porque escucho canciones" como se muestra en el ejemplo del dibujo que ilustra una pequeña conversación. Se pide a los alumnos que hagan uso de la lista de actividades, además de un recuadro con las conjugaciones del verbo hacer, que pueden servir de guía para conjugar los verbos en la lista. Como vemos, este tipo de ejercicio está enfocado en las conjugaciones y posiblemente en el tipo de estructura usando el conector "porque" o la pregunta "por qué" además del vocabulario de actividades relacionadas con actividades escolares. A continuación se explican los pasos del proceso creativo a partir de este ejemplo de ejercicio los cuales son presentados con sus respectivas preguntas guía en la Tabla 1.

Paso 1. Analizar el input. Este primer paso implica observar el material por el libro para determinar el objetivo del ejercicio (Ellis, 2003) así como el input dado al alumno para completar el ejercicio (v.g. el tema, la situación, el ejemplo, el contexto, etc.). Las instrucciones mismas del ejercicio son un punto de partida para revisar si existen textos o ejemplos del tipo de actividad por realizar. En el caso del ejemplo, el objetivo principal es expresar nuestros gustos. Para ello se hace uso del tema de las asignaturas académicas, pero lo que se le pide hacer al alumno es simplemente escribir oraciones. Este tipo de actividad no está vinculada con la vida real dado que podemos preguntarnos: ¿para qué voy a expresar mis gustos? ¿Por qué de manera escrita? ¿Quién los puede leer? Estas preguntas tienen muchas posibles respuestas, pero no están dadas en ninguna parte del ejercicio. Por ahora, nos conformamos en ver que el input ofrecido al alumno son dos modelos de lenguaje en uso, uno de pregunta: “¿Por qué te gusta el inglés?" y el otro da una respuesta con justificación: "Me gusta el inglés porque escucho canciones". Además de eso, está la lista de otras actividades académicas, con lo cual podemos decir que existe un input básico pero suficiente para poder desarrollar una tarea. En cuanto a si es o no atractivo para los alumnos, depende de 
el grupo y del conocimiento que el docente tenga del mismo. Por tanto, este ejercicio tiene un objetivo lingüístico y el suficiente input para que los alumnos lo desarrollen. Dados los conceptos de tarea de ensayo o de activación de Nunan (2004) y el objetivo como lo establece Ellis (2003), es necesario hacer una conexión entre este tipo de uso del lenguaje y un contexto de la vida real, lo cual se realiza en el siguiente paso.

Paso 2. Pensar en un contexto de la vida real. Para que una tarea sea efectiva, es importante su vinculación con la vida real. Sabemos que el objetivo lingüístico es hacer uso correcto del vocabulario y las estructuras; entonces se le pide al alumno puntualmente escribir sobre sus gustos académicos. Retomando la pregunta: "¿Para qué voy a expresar mis gustos?", queda claro que es muy común tener que expresar nuestros gustos en diversas actividades sociales (e.g. conversaciones con amigos, perfiles de redes sociales, cuestionarios de personalidad de revistas, relaciones amorosas, etc.). La forma para expresar este tipo de conceptos aplica fácilmente para un medio oral o escrito, por lo que podemos decidir.

Sin embargo, no es solo el hecho de que se pueda dar en la vida real, sino en detectar cuál es el objetivo comunicativo de hacerlo en cada una de esos contextos. Por ejemplo, si es una conversación con amigos o una red social, mi objetivo probablemen- te es encontrar puntos de vista similares o diversos sobre alguna de estas actividades e incrementar mi número de amigos o participar en diversos clubes o grupos en línea. Por otra parte, un cuestionario de personalidad de revista es algo que las personas pueden hacer en la cotidianidad, pero también puede darse de una forma más rigurosa y seria, por ejemplo, como una guía vocacional para vislumbrar futuros trabajos. En cualquier caso, no basta con decirle a los alumnos: "escribe tus gustos como si lo hicieras en una red social" sino que se le debe dar un objetivo, por ejemplo, "Escribe tus gustos y luego busca a quienes tienen tus mismos gustos" (tarea enfocada, Ellis, 2003) o mejor aún "una vez que escribas lo que te gusta y lo que no, decide con tu compañero cuál de esas actividades pueden realizar juntos durante la semana" (tarea desenfocada, Ellis, 2003).

Así, un simple listado de actividades educativas se convierte en un proceso de socialización que le da más sentido al acto por realizar. Parte de dar un contexto claro, radica en dar roles específicos para los alumnos. Si tomamos por ejemplo el caso de una relación amorosa, podemos decirles: "estás buscando pareja, encuentra a alguien que tenga tus mismos gustos, luego decide si puede o no ser tu futura pareja". Así queda implícito el rol de cada estudiante a la vez que se les da un objetivo claro y auténtico.

Figura 1. Ejemplo de un ejercicio tradicional de libro de texto para enseñanza del español.

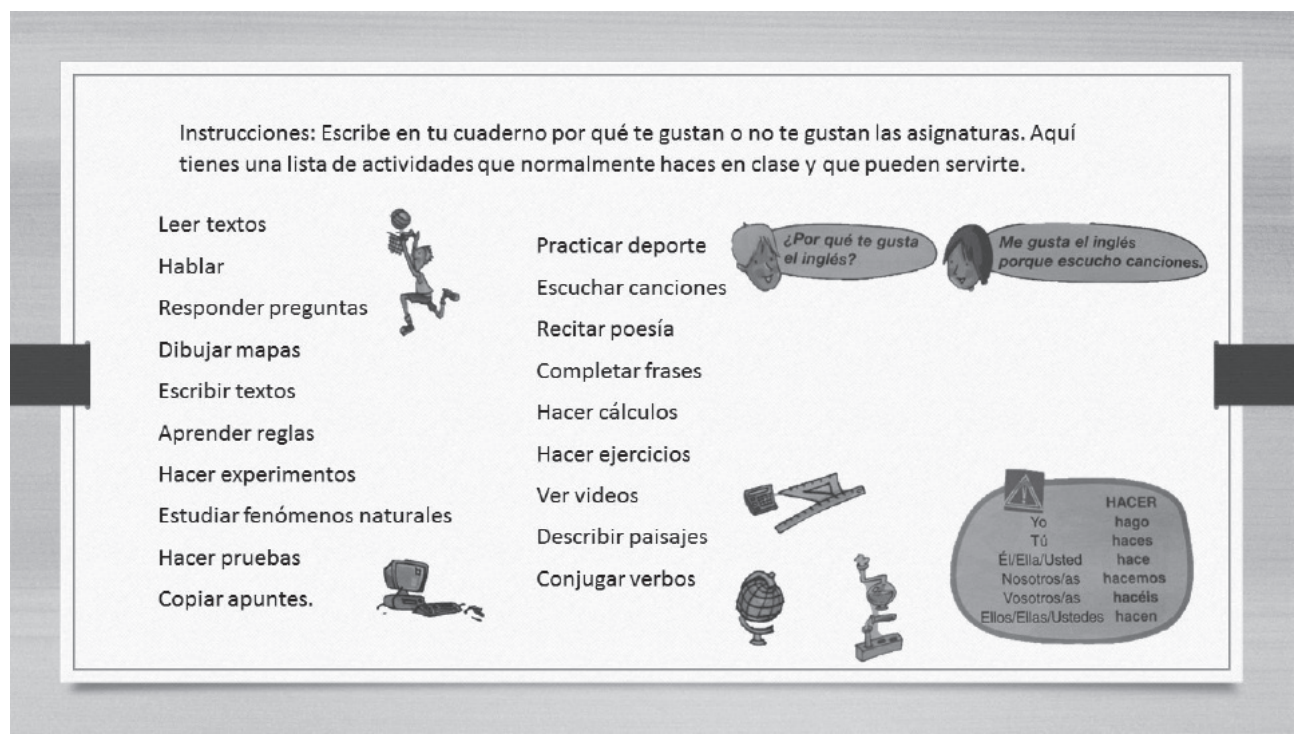

Adaptado de Vera \& Salamone (2007). 
Determinar si se utiliza una tarea de ensayo (uso de redes sociales) o de activación (test vocacional, asumiendo que los alumnos no realizan este tipo de test) puede ayudarnos a darles variedad a las tareas escogidas haciendo que algunas estén más centradas en la realidad, mientras otras estimulen la parte lúdica. Cada una de los posibles contextos y objetivos trae no solo el uso del lenguaje meta, sino que ofrece pequeñas variaciones. Por ejemplo, no es el mismo tipo de registro en cada caso. Esto es una de las características del uso de un aprendizaje por tareas: la amplitud de posibilidades de uso de lenguaje que se da. Por este motivo, se debe hacer un análisis de los tipos de lenguaje que entran en juego en el proceso de realizar la tarea.

Paso 3. Lenguaje en la situación y Paso 4. Lenguaje en clase (para negociar). Hay que tener en cuenta la existencia de dos tipos de lenguaje que los alumnos necesitan para llevar a cabo la tarea. Uno es el lenguaje de la situación, el requerido para llevar a cabo el objetivo comunicativo en la vida real y el otro, el lenguaje en clase: todos los pequeños intercambios comunicativos que no están relacionados con la situación de la vida real pero que se necesitan para, por ejemplo, hacer consultas al docente u organizar la actividad. De esta forma, el lenguaje de la situación en el ejercicio de ejemplo pueden ser dos evidentes: a) la formación de preguntas y respuestas con "por qué" y "porque" y b) la conjugación de los verbos según el paradigma.

No obstante, este no es el único lenguaje meta posible. A pesar de que una tarea puede ser enfocada o desenfocada (Ellis, 2003), es muy común que las necesidades lingüísticas de los alumnos sobrepasen los límites planteados. Si vemos el ejercicio de ejemplo, puede que los alumnos tengan dudas en el manejo de las tildes o en la pronunciación de segmentos o supra segmentos. Además, cabe la posibilidad de que el alumno haga uso de otras estructuras más allá de las esperadas del estilo "a mí me gusta describir paisajes en clase", en donde no se da la conjugación del verbo "describir" como está planteado en el ejercicio. Estar un paso delante de este tipo de situaciones le permite al docente responder con mayor eficacia las dudas que puedan surgir. Por lo demás, en el enfoque por tareas, este tipo de uso se considera beneficioso para el alumno, pues estimula un uso del lenguaje y un proceso de adquisición más natural, dirigido por sus aptitudes y necesidades y no por un objetivo lingüístico inmutable.

Por su parte, el lenguaje en clase es aquel que el alumno va a utilizar para llevar a cabo la tarea en clase. Ya sea en la interacción con el docente o entre compañeros, el alumno puede necesitar una aclaración de las instrucciones o preguntas puntuales sobre el manejo de la clase, la actividad o el sentido de lo que hace. En nuestro ejemplo, se pueden esperar negociaciones del tipo: "yo hago esta parte primero y tú la otra" o "habla más duro que no te entiendo". También existen frases útiles para favorecer la autorregulación de su aprendizaje como: "creo que ese no se conjuga así" o "¿estás seguro de la pronunciación?". Hobbs (2012) llevó a cabo un interesante estudio con hablantes nativos del inglés a quienes les pidió realizar tareas diseñadas para alumnos de inglés como lengua extranjera con el fin de examinar el tipo de lenguaje que estos usan y levantar un mini corpus. A la fecha, el autor no tiene noticia de algún trabajo de este estilo en español, por lo que se recomienda hacerlo.

Paso 5. Resultado esperado. De la mano con el objetivo, una tarea debe ser clara en cuanto al tipo de resultado esperado. Una vez más, la pregunta no es si el alumno tuvo o no errores de tipo lingüístico (es esperable y deseable que los tengan) sino sobre si consiguió completar la tarea. Tanto el docente como los alumnos deben tener claro cuándo se ha terminado el acto comunicativo (resultado) y la forma que este debe tomar ya sea escrito u oral (proceso). Para ello es primordial la escogencia del verbo en las instrucciones. Por ejemplo, se le pide a un grupo de estudiantes simplemente: "discutan sobre sus gustos", ellos no tendrán claro qué tanto deben discutir, ni sabrán cuándo pueden o no dejar de discutir. Los participantes pueden caer en apatía pues sin un propósito para conversar o escribir, estos actos carecen de valor. En vez de eso, es preferible pedirles llegar a una decisión o concretar alguna idea, por ejemplo: "escucha las ideas de tus compañeros y escoge con quien compartes más gustos", o "crea un club con las personas que tienes al lado, escoge las actividades que van a realizar en ese club de acuerdo con los gustos de cada quien". En este sentido, se da por hecho la discusión hecha por los alumnos, pero su 
objetivo está más allá de usar palabras y transmitir ideas al pedírseles que tomen una decisión. Además, se da una razón para la escucha atenta de las ideas de los compañeros. Otros verbos que son útiles pueden ser: "persuada", "convenza", "seleccione", pero uno de los que se adapta más fácilmente a casi cualquier contexto es "decida". La escogencia del tipo de verbo para el objetivo responde con claridad a la vida real, que se reflejan en los tipos de tareas dados por Pica, Kanagy y Falodun (1993) y Willis (1996).

En otro tipo de actividad, por ejemplo en presentaciones orales, se les puede pedir a la audiencia que tome notas o haga preguntas con el fin de garantizar su atención. Este tipo de resultados esperados, reflejados en los verbos del objetivo, van de la mano con el contexto, la situación y los participantes que se hayan definido anteriormente y, sobretodo, con los resultados obtenidos de la misma actividad comunicativa pero en la vida real. Cuando estos resultados estén claros, la evaluación de la actividad estará clara, ya que esta consistirá en primera instancia si se logró o no terminar con la tarea propuesta y, en términos lingüísticos, la medida en que el uso del lenguaje facilitó o no la tarea. La evaluación se convierte en una retroalimentación en la cual la corrección iría en el sentido "es más

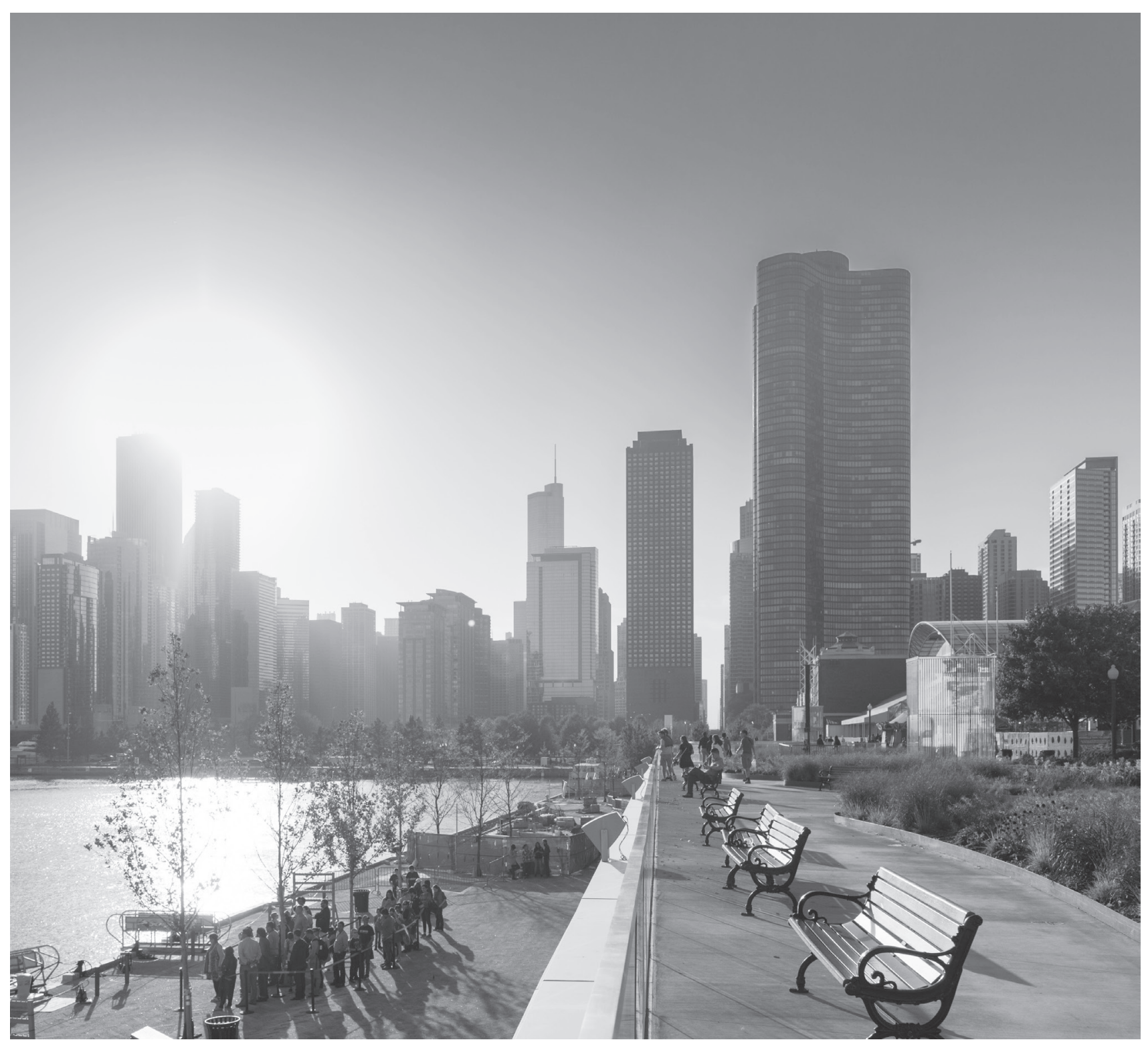

Fotografía de Leonardo Corrales N. 
efectivo si lo haces de esta forma" y no en el de uso "correcto" o "incorrecto" del lenguaje.

\section{Tabla 1}

\section{Proceso creativo para transformar ejercicios en tareas comunicativas}

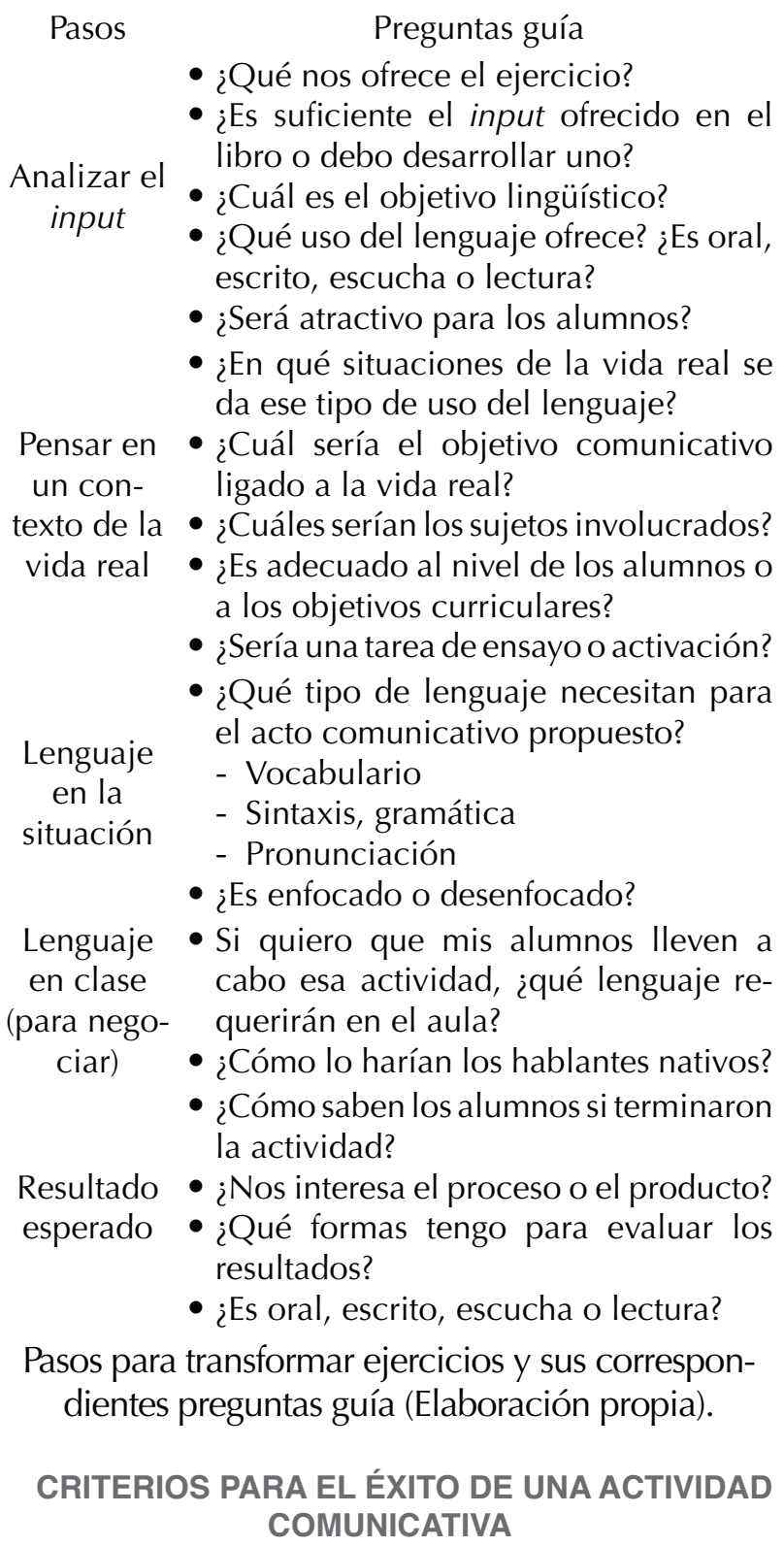

Muchos son los criterios dados por distintos autores para determinar la efectividad de una tarea (Ellis, 2003; Hérnandez, 2006; Cirocki y Widodo, 2012). La tabla 2 ofrece un condesado de algunos de estos criterios los cuales, una vez terminado el diseño de la tarea, pueden servir de guía para determinar la eficacia de lo creado. No son todos los criterios son posibles, ya que mucho de eso depende del contexto sociocultural así como de las particularidades de los grupos, docentes y alumnos. Sin embargo, se ofrece como guía y se recomienda a los docentes acrecentar la lista y discutir con pares la pertinencia de los nuevos criterios. Para ello, existe un criterio general (Ellis, 2003) que es el que puede guiar este proceso: una tarea es exitosa si resulta en una comunicación enfocada en el contenido. Puede no serlo si resulta en un despliegue de conocimiento lingüístico por parte del alumno.

Esta consideración final sobre los criterios debe estar siempre presente si se quiere hacer uso de tareas en clase, y sobre todo, si se quiere diseñar tareas a partir de los ejercicios.

\section{Tabla 2}

\section{Criterios para evaluar la efectividad de una tarea}

\section{Criterios}

1 La actividad resulta en una comunicación centrada en el contenido.

La actividad es auténtica (el lenguaje usado

2 corresponde a una conducta comunicativa del mundo real).

3 La actividad es observable y puede ser juzgada en términos de su contenido.

4 Lo realizado por los alumnos refleja las intenciones del instructor.

5 El tiempo dado es suficiente para llevar a cabo la actividad.

Los materiales (input) son adecuados o suficientes para que el alumno lleve a cabo la tarea solicitada. Existe una relación clara entre el input y lo que se espera del alumno.

7 La actividad se enfoca en una habilidad (oral, escucha, escrita, lectura) o en la unión de estas.

8 Existe un proceso cognitivo como clasificar, razonar, enlistar, seleccionar, etc.

9 La actividad puede ser fácilmente evaluable.

10 Los estudiantes están motivados.

Los criterios son un condesado tomado principalmente de Ellis, 2003 y Hérnandez, 2006. 


\section{CONCLUSIONES}

El aprendizaje por tareas es un enfoque comunicativo que plantea una filosofía del aprendizaje así como una metodología y estructuración de la clase basada en el concepto de tarea. En este trabajo se presentó una forma de usar los principios de ese enfoque para transformar ejercicios tradicionales en tareas comunicativas. El propósito de dicha transformación es ofrecer a los docentes una herramienta metodológica en forma de pasos que les permita hacer un uso creativo de algunos de los ejercicios tradicionales para darle variedad y mayor efectividad a los procesos de adquisición de una lengua. Lo más importante de este proceso es no olvidar que, dentro de un enfoque comunicativo, prima el contenido y el propósito de la comunicación, para lo cual la forma, ya sea gramática, vocabulario etc., ayudan a la vez que son adquiridos de manera natural. No se pretender desvirtuar la importancia de los ejercicios enfocados en la forma ya que estos son necesarios como proceso de consolidación del aprendizaje, pero sí se quiere ayudar a los docentes a hacer un uso más variado y creativo de los materiales didácticos con los que cuentan.

\section{REFERENCIAS:}

Cirocki, A. y Widodo, H. (2012). Innovation and Creativity in ELT Methodology. New York: Nova Science Publishers, Inc.

Ellis, R. (2003). Task-based language learning and teaching. New York, NY: Oxford University Press.

Ellis, R. (2009). Task-based language teaching: sorting out the misunderstandings. International Journal of Applied Linguistics. 19(3), 221- 246.

Fernández López, S. y Navarro Blanco, A. (2010) Enfoque por tareas: propuesta didáctica. En Lluch A. (Coordinador), 2010, Colección complementos serie didáctica. Brasilia DF: Secretaría general técnica, Editorial.

Hernández, A. (2006). Characteristics of successful tasks which promote oral communication. Comunicación, 15(1), 46-56.

Hobbs. J. (2012). Task structure and patterns of interaction. What can we learn from observing native speakers performing tasks? En Coombe, C. y Shehadeh, A. (2012). Task-based Language
Teaching in Foreign Language Contexts: Research and Implementation.Philadelphia: John Benjamins Publishing, 109-136.

Lázaro, O. (1999). La enseñanza mediante tareas. A cien años del 98 lengua española, literatura y traducción: actas del XXXIII Congreso Internacional de la Asociación Europea de Profesores de Español / Manuel Ramiro Valderrama (aut.), 1999, 295-310.

Lee, J. (2000). Tasks and communicating in language classroom. Mexico City: McGraw-Hill companies.

Martín, E. (2009). El enfoque por tareas y la didáctica en lengua inglesa en el nuevo Grado de Magisterio. Didáctica. Lengua y Literatura (21), 255-279.

Nunan, D. (1989). Designing tasks for the communicative classroom. Cambridge: Cambridge University Press.

Nunan, D. (2004). Task-based language teaching. Cambridge: Cambridge University Press.

Pica, T., Kanagy, R. y Falodun, J. (1993). Choosing and using communicative tasks for second languaje. England: MM.

Richards, J.C. y Rodgers, T.S. (2001). Approaches and methods in language teaching. New York, NY: Cambridge University Press.

Robertson, M. (2014). Task-Based Language Teaching and Expansive Learning Theory. TESL CANADA JOURNAL (31), 187-198.

Rodríguez-Bonces M. \& Rodríguez-Bonces J. (2010) Task-Based Language Learning: Old Approach, New Style. A New Lesson to Learn. PROFILE, (12), 165-178.

Vera, A. \& Salamone, R. (2007). Paso a Paso. Brugherio: Modern Publishing House.

Willis, J. (1996). A framework for Task-Based learning. Addison Wesley Longman Lt

Willis, D., \& Willis, J. (2012). Doing task-based teaching. Oxford, UK: Oxford University Press.

Xerri, D. y Vassallo, O. (2016). Creativity in ELT: an introduction. En Xerri, D y Vassallo, O. (ed.). (2016). Creativity in English Language Teaching. ELT Council, 1-8.

Zanón, J. (1995). La enseñanza de las lenguas extranjeras mediante tareas. Signos. Teoría y práctica de la educación (14), 52-67. 